\author{
(О Е.Н. Андреева ${ }^{1,2}$, Ю.С. Абсатарова'*, Е.В. Шереметьева', В.А. Фурсенко
}

'ФГБУ «Национальный медицинский исследовательский центр эндокринологии» Минздрава России, Москва, Россия ${ }^{2}$ ФГБОУ ВО «Московский государственный медико-стоматологический университет им. А.И. Евдокимова» Минздрава России, Москва, Россия

В статье представлены данные о последствиях ожирения для женской репродуктивной системы и потомства матерей с избыточной массой тела или ожирением, таких как бесплодие, выкидыши, преждевременные роды, мертворождение, врожденные аномалии и недоношенность, а также высокий риск кесарева сечения. Ожирение сопутствует синдрому поликистозных яичников, ухудшая метаболический профиль и повышая риск развития депрессии и расстройства пищевого поведения. Ожирение и гипергликемия матери способны оказывать влияние на формирование плода путем эпигенетических механизмов без затрагивания нуклеотидных последовательностей. В дальнейшем у потомков матерей с ожирением или избыточной массой тела и гестационным сахарным диабетом повышаются метаболические и сердечно-сосудистые риски. Для пациенток с ожирением характерен дефицит фолиевой кислоты и недостаточность лютеиновой фазы. Экзогенное введение этих веществ способствует улучшению исходов беременности и профилактирует врожденные пороки развития.

КЛЮЧЕВЫЕ СЛОВА: ожирение, избыточная масса тела, синдром поликистозных яичников, гестационный сахарный диабет, эпигенетика, внутриутробное программирование, дисфункция яичников.

\title{
OBESITY AND REPRODUCTIVE FUNCTION OF WOMEN: EPIGENETIC AND SOMATO-PSYCHOLOGICAL FEATURES
}

\author{
(c) Elena N. Andreeva ${ }^{1,2}$, Yulia S. Absatarova ${ }^{1 *}$, Ekaterina V. Sheremetyeva' ${ }^{1}$ Valentina A. Fursenko ${ }^{1}$
}

'Endocrinology Research Centre, Moscow, Russia

${ }^{2}$ A.I. Yevdokimov Moscow State University of Medicine and Dentistry, Moscow, Russia

The review article presents data on the effects of obesity on the female reproductive system and offspring of mothers with overweight or obesity, such as infertility, miscarriages, premature birth, stillbirth, congenital anomalies and prematurity, as well as a high risk of cesarean section. Obesity accompanies polycystic ovary syndrome, worsening the metabolic profile and increasing the risk of developing depression and eating disorders. Maternal obesity and hyperglycemia are able to influence the formation of the fetus by epigenetic mechanisms without affecting the nucleotide sequences. Subsequently, the metabolic and cardiovascular risks increase in the descendants of obese or overweight mothers and gestational diabetes. Patients with obesity are characterized by a folic acid deficiency and a deficiency of the luteal phase. Exogenous administration of these substances improves pregnancy outcomes and prevents congenital malformations.

KEYWORDS: obesity, overweight, polycystic ovary syndrome, gestational diabetes, epigenetics, intrauterine programming, ovarian dysfunction.

Ожирение стало одной из глобальных эпидемий, охватывающей более 600 миллионов человек во всем мире. ВОЗ сообщает, что в 2014 году 39\% взрослых старше 18 лет имели избыточный вес, а 13\% страдали ожирением, 41 миллион детей в возрасте до 5 лет страдали избыточным весом или ожирением [1]. Согласно данным BO3, стандартизированная по возрасту распространённость ожирения среди женщин в возрасте 18 лет и старше составила $26,9 \%$ [2].

При этом важно отметить, что число людей с лишним весом постоянно растёт. Так, если в 2006 году ожирение имели 20,4\% россиян, то в 2016 году - уже 23,1\%. Женщин с ожирением примерно на 8-9\% больше, чем мужчин [2].

Избыточный вес и ожирение связаны с неблагоприятными последствиями для женской репродуктивной системы, такими как бесплодие, выкидыши, преждевременные роды, мертворождение, врожденные аномалии и недоношенность, а также высокий риск кесарева сече- ния, плохое заживление ран, более короткая продолжительность грудного вскармливания и депрессия [3].

Чрезвычайно велико влияние ожирения, оказываемое на репродуктивную способность женщины через функциональные изменения гипоталамо-гипофизарно-яичниковой оси. У больных с избыточной массой тела и ожирением повышается уровень инсулина, который стимулирует синтез андрогенов в яичниках. Из-за высокого процента жировой ткани андрогены ароматизируются в эстрогены, а это в свою очередь по принципу отрицательной обратной связи влияет на гипоталамо-гипофизарнояичниковую ось и производство гонадотропинов [3]. Это проявляется менструальной и овуляторной дисфункцией. Гиперинсулинемия является звеном патогенеза синдрома поликистозных яичников (СПЯ). Ожирение способствует снижению чувствительности тканей к инсулину и усугубляет симптомы СПЯ, манифестируя более тяжелым фенотипом. При гиперандрогении усиливается накопление 
висцерального жира, что проявляется резистентностью к инсулину и гиперинсулинемией, тем самым активизируется производство овариальных и надпочечниковых андрогенов и замыкается порочный круг [4].

СПЯ затрагивает около 5-10\% женщин репродуктивного возраста. Основными компонентами синдрома являются овуляторная дисфункция, гиперандрогения и увеличение объемов яичников [5]. Для пациенток с СПЯ также характерны нарушения психического здоровья, такие как депрессия и тревога, что приводит к снижению психосоциального благополучия женщины, сексуального удовлетворения и общего качества жизни [6]. Больные СПЯ меньше удовлетворены своим телом по сравнению со здоровыми женщинами. По разным оценкам до 75\% пациенток с СПЯ имеют ожирение, хотя распространенность варьирует в зависимости от географического региона. Расстройства настроения, неудовлетворенность телом, несомненно, являются ключевыми факторами в формировании качества жизни и связаны с отношением к еде и общими проблемами питания [7].

Для больных СПЯ характерно нарушение пищевого поведения [8] и более высокое потребление углеводов [9]. Была установлена связь между гиперандрогенией и поведенческими нарушениями, такими как импульсивность, что делает женщин с СПЯ более подверженными к булимии. Это, вероятно, связано с повышенной неудовлетворенностью своим телом, что побуждает пациенток к более экстремальному диетическому контролю, тем самым увеличивая риск развития неупорядоченного пищевого поведения [10]. Программы контроля веса, предлагаемые больным СПЯ, часто направлены на ограничение калорий и увеличение физической активности. В результате модификации образа жизни и потере веса всего на 5-10\% от первоначальной массы имеют положительную динамику многие клинические и лабораторные показатели [11]: уменьшается интенсивность гиперандрогении [12], становится менее выражена резистентность к инсулину [12], уменьшаются уровни атерогенных фракций липидов [13], становится более регулярным менструальный цикл [11], появляются спонтанные овуляторные менструальные циклы и увеличивается процент наступления беременности $[14,15]$ и улучшаются показатели психологического статуса $[11,68]$. Кроме того, высокие уровни андрогенов у женщин с СПЯ обусловливают большую тягу к жирным и углеводистым продуктам, что дополнительно препятствует снижению веса. Для повышения сытости на фоне низкокалорийной диеты рекомендуют увеличить потребление белка, но высокобелковая диета не приводят к более высокой потере веса или более значительному улучшению метаболических симптомов по сравнению с низкобелковыми диетами [16]. По данным недавнего исследования диета для пациентов с гипертензией (включающая фрукты, овощи, цельные зерна, молочные продукты с пониженным содержанием жира, холестерина) приводит к сопоставимой потере веса при сравнении со стандартной низкокалорийной диетой у женщин с СПЯ и значительным улучшениям чувствительности к инсулину, показателям глобулина, связывающего половые гормоны, и антимюллерова гормона [12]. Учитывая, что больные СПЯ находятся в группе риска расстройства поведения и беспорядочного питания, важно включать в алгоритм ведения консультацию психоневролога.
У женщин репродуктивного возраста с ожирением по данным многочисленных исследований увеличивается промежуток от момента планирования беременности до зачатия. Согласно результатам крупного исследования по мере увеличения индекса массы тела (ИМТ) наблюдалось снижение коэффициента фертильности [17]. Интересным представляется факт о том, что несмотря на отсутствие овуляторной дисфункции, женщины с ожирением могут быть субфертильными. Изучение крупной американской когорты из более чем 7000 женщин показало, что у женщин с избыточной массой тела или ожирением и регулярным менструальным циклом снижается фертильность, а в большой голландской когорте из более чем 3000 женщин с нормальным циклом вероятность спонтанной беременности снижалась линейно при увеличении ИМТ> 29 кг/м² [15, 17].

Ожирение влияет на исходы вспомогательных репродуктивных технологий (ВРТ) не только за счет овуляторной дисфункции. Пациентки с ожирением, вступившие в протокол экстракорпорального оплодотворения, имеют меньшее количество ооцитов, которые потенциально могут быть оплодотворены [18]. Исследования продемонстрировали связь ожирения и показателей живорождения в зависимости от увеличения ИМТ. Наличие морбидного ожирения (ИМТ>40 кг/м²) на 50\% снижает вероятность живорождения [19].

В Институте медицины (Вашингтон, США) были разработаны рекомендации по набору массы тела во время беременности на основе догестационного ИМТ [20]. Женщины с недостаточным и нормальным весом могут прибавить 12,7-18,1 и 11,3-15,9 кг соответственно, тогда как у пациентки с избыточным весом и ожирением 6,8-11,3 и 5-9 кг соответственно. Чрезмерная прибавка веса происходит в 41-74\% беременностей [21]. При этом те, у кого был избыточный вес или ожирение до зачатия, имеют повышенный риск чрезмерного гестационного набора веса [22]. Активно изучается связь между неадекватной прибавкой массы тела у женщин с избыточным весом и ожирением и последствиями для матери и плода. В исследовании, проведенном Zilko и соавт., прибавка меньше рекомендуемых 6,8 кг была связана с более низким риском преэклампсии, кесарева сечения и макросомии [23].

Отрицательная самооценка своего тела женщиной может иметь пагубные последствия (диетические погрешности, нездоровое питание и депрессию), что приводит к увеличению риска преждевременных родов и послеродовой депрессии [24]. По данным Skouteris и соавт. женщины наиболее не удовлетворены и не способны адаптироваться к изменениям в своем теле, возникающим в начале и середине второго триместра [25].

Ожирение имеет неблагоприятные последствия для здоровья как матери, так и ее ребенка путем увеличения риска ожирения у потомства [26]. На сегодняшний день процессы, ответственные за программирование плода, еще не полностью изучены. Waddington C.Н., британский биолог, первым ввел термин «эпигенетика». В 1942 году он использовал этот термин для определения результата взаимодействия между генами и окружающей средой при установлении фенотипа [27]. Эпигенетика изучает механизмы развития признаков, которые наследуются клетками потомства, хотя они не связаны с мутациями нуклеотидной последовательности [28]. 
Избыточная масса тела матери способствует развитию макросомии плода и повышенному содержанию жировой ткани [29]. Новорожденные предрасположены к ожирению в детстве и сахарному диабету 2 типа и сердечно-сосудистым заболеваниям во взрослой жизни [30]. Таким образом, внутриутробная среда (которая зависит от питания матерей) регулирует не только риск заболевания, но также момент его возникновения и интенсивности патологического состояния.

Конкретные механизмы, ответственные за эти связи, пока не обнаружены. Однако есть предположения о роли системного метаболизма и изменений иммунологической системы в организме, подверженном неблагоприятным факторам внутриутробной среды. Одна из теорий внутриутробного программирования предполагает, что гиперкалорийная диета во время беременности приводит к нарушению функции инфундибулярного ядра гипоталамуса, контролирующего потребление пищи через развитие резистентности к лептину (гормон, который регулирует энергетический гомеостаз путем подавления ощущения голода) [31]. Исследования, проведенные Guenard и соавт., свидетельствуют о положительной корреляции между ИМТ, высоким уровнем глюкозы, общего холестерина и липопротеинов низкой плотности в сыворотке крови матери и метаболическим синдромом, и возникновением кардиометаболического синдрома у потомства [32]. Эту связь можно объяснить тем, что гиперкалорийная диета во время беременности, сопровождающаяся нарушениями обмена веществ, приводит к гиперлипидемии, гипергликемии и гиперинсулинемии, которые оказывают неблагоприятное влияние на развитие и функцию плаценты [30]. Поскольку плацента отвечает за обеспечение питательными веществами плода, в сумме эти неблагоприятные эффекты оказывают синергетическое действие на потомство, способствуя реализации отрицательных эпигенетических исходов. Дети матерей, употреблявших большое количество жиров во время беременности, склонны к набору лишнего веса. Считается, что в этом случае дефицит адипонектина (гормона, ответственного за чувствительность к инсулину) является фактором, инициирующим эпигенетические модификации [33]. Диета с высоким содержанием жиров во время беременности инициирует недостаточную сосудорасширяющую реакцию на ацетилхолин у потомства. Это приводит к существенному нарушению основной функции эндотелия и предрасположенности к развитию гипертонии и тяжелым сердечно-сосудистым заболеваниям [34].

Гестационный сахарный диабет (ГСД) и сопутствующая гипергликемия, независимо от материнского ожирения, являются важными факторами, определяющими внутриутробное программирование. Факторы транскрипции в бета-клетках инсулина поджелудочной железы чрезвычайно чувствительны к субоптимальной внутриутробной среде. Было замечено, что гипергликемия является основным участником эпигенетических модификаций и повышает риск развития нарушения толерантности к глюкозе у потомства [35]. Метаболические нарушения, связанные с ГСД, способствуют накоплению активных форм кислорода, которые возникают из-за дефицита антиоксидантов не только в организме матери, но и плода. Это приводит к отдаленным последствиям, таким как нарушение энергетического гомеостаза, развитие ожирения в детском возрасте и раннее начало сахарного диабета типа 2 у потомства [36]. Согласно Marchi и соавт. риск развития ГСД в 4 раза выше среди женщин с ожирением и в 10 раз выше среди женщин с тяжелым ожирением (ИМТ от 33 до 40 кг/м²), по сравнению с беременными женщинами с нормальным весом [37].

Уменьшенная длина теломер в развивающемся организме является одним из подтвержденных механизмов внутриутробного программирования, инициированным ГСД [38]. Была обнаружена связь между упомянутыми выше изменениями и высоким риском сердечно-сосудистых заболеваний во взрослой жизни [39].

Эпигенетические механизмы необходимы для правильного развития млекопитающих. Большинство эпигенетических вариантов становятся невозможным в начале эмбрионального развития (стадии бластоцисты) [40]. Большинство процессов, связанных с дифференцировкой клеток, инициируются и поддерживаются посредством эпигенетических изменений. Те процессы, которые происходят во время разделения клеток, обычно удаляются в зародышевой линии. Существует гипотеза, что эффекты программирования могут также наблюдаться в нескольких поколениях после специфических эпигенетических изменений даже при оптимальной доступности питательных веществ. Это позволяет распознавать эпигенетические механизмы как основной метод передачи фенотипа родителей потомству и последующим поколениям. Материнский метаболический синдром рассматривается как инициирующий фактор эпигенетических изменений в клетках плода, что может свидетельствовать о межпоколенческом наследовании предрасположенности к метаболическим и сердечно-сосудистым заболеваниям [41].

Адекватное питание во время беременности может не только улучшить здоровье матери, но и привести к значительному снижению риска развития сахарного диабета и профилактике детского ожирения. Исследования, проведенные на животных моделях, свидетельствуют о том, что низкобелковая диета может в определенных условиях приводить к повышенной чувствительности плода к инсулину [42]. Таким образом, правильно подобранная диета во время беременности может способствовать созданию нового, «более здорового» эпигенетического шаблона и устранению неблагоприятных последствий. Было признано, что сбалансированная средиземноморская диета значительно снижает уровень глюкозы, липидов и липопротеинов в сыворотке крови матери и эмбриональной крови, и снижает риск ГСД и низкого веса при рождении [43].

Исследования, проведенные наживотных, предполагают возможность сдерживания эпигенетических механизмов и предотвращения побочных эффектов, вызванных внутриутробным программированием, при оптимальном наличии питательных веществ. Lillycrop и соавт. обнаружили полезные и защитные эффекты фолиевой кислоты наряду с низкобелковой диетой во время беременности [44].

Дефекты нервной трубки (ДНТ), известные последствия дефицита фолиевой кислоты, являются второй наиболее распространенной причиной серьезных врожденных пороков с частотой 2-10 детей на 1000 беременностей во всем мире [45]. Недостаточное потребление фолатов с пищей - одна из ведущих причин дефицита этой кислоты [46]. Другими причинами являются повышенная 
потребность в связи с беременностью или неопластическими заболеваниями, мальабсорбция, лекарственные препараты, антагонисты фолиевой кислоты или другие ингибиторы обмена веществ, употребление алкоголя (что влияет как на потребление фолата, так и на абсорбцию). Исследования среди женщин репродуктивного возраста демонстрирует обратную связь ИМТ и уровня фолиевой кислоты в крови [47], и довольно часто эта связь не зависит от диеты, возраста, приема витаминных добавок и паритета [48].

Примерно одна треть беременностей во всем мире является незапланированной, и многие могут не знать о своей беременности в течение нескольких недель после зачатия, поэтому рекомендуется использовать фолиевую кислоту всем женщинам репродуктивного возраста, особенно тем, кто страдает от избыточного веса или ожирение [49]. Крайне важно принимать фолиевую кислоту в периконцепционный период, по крайней мере, за три месяца до беременности [50]. На сегодняшний день существуют комбинированные оральные контрацептивы (KOK) с дроспиреноном и дополнительным содержанием фолатов, что позволит решить 2 задачи - контрацепция и подготовка к беременности. Исследования показали, что обогащенный фолиевой KOK значительно увеличивал концентрацию фолата в крови в течение 24 недель [51]. Целевая аудитория этих препаратов - наиболее нуждающиеся в фолиевой кислоте женщины детородного возраста, и данная стратегия имеет огромные преимущества в отличие от широкого применения диетического обогащения фолатами. Таким образом, в случае незапланированной беременности КОК с фолатами обеспечивает их оптимальный уровень для профилактики ДНТ [52]. Независимо от того, планирует женщина беременность или нет, препараты обеспечивают необходимой ежедневной дозой фолата для поддержания здоровья.

У пациенток с ожирением часто имеется неполноценность лютеиновой фазы, при которой недостаточно синтезируется эндогенный прогестерон для поддержания секреторного эндометрия, имплантации и роста эмбриона. Противоречивость клинического значения недостаточности лютеиновой фазы объясняется отчасти отсутствием надежного теста для диагностики этого расстройства. Она также характерна для бесплодия, СПЯ и выкидышей $[11,53]$. У женщин с ожирением происходит уменьшение амплитуды импульса лютеинизирующего гормона (ЛГ), и наблюдается значительное снижение экскреции глюкуронида прегнандиола (основного метаболита прогестерона) в лютеиновой фазе по сравнению с группой контроля нормального веса [54]. Однако стоит подчеркнуть, что репродуктивные потери - это моноили, как правило, поликомпонентное расстройство, содержащие в себе: воспалительный генез, гормональными нарушения (в основном, конечно же прогестерон-дефицит), децидуальное старение и сосудистые аномалии, которые могут негативно проявить себя на этапе от предгравидарной подготовки до послеродового периода.

Пациенты с эндокринопатиями, к которым относится как ожирение, так и СПЯ, должны относиться к пациентам с «высоким риском» в прогнозе развития «неправильного» течения беременности $[11,55]$. Синдром гиперандрогении, избыточная масса тела/ожирение, инсулинорези- стентность, «паразитарный» уровень ЛГ, недостаточность лютеиновой фазы (2ой фазы), гиперпролактинемия, гипотиреоз/тиреотоксикоз являются эндокринными факторами невынашивания беременности [56]. Спонтанная потеря плода происходит у 40\% женщин с СПЯ, но основной причиной этого, как правило, является не само заболевание, а ожирение; гиперинсулинемия, инсулинорезистентность; гипергомоцистеинемия; высокий уровень фактора ингибитора-активатора плазминогена-1; низкая рецептивность эндометрия; «паразитарный» уровень ЛГ [57-62].

Дефицит прогестерона у женщин с ожирением и метаболическим синдромом может приводить к нарушению процессов инвазии трофобласта, к нарушению имплантации, а, следовательно, и синдрому потери плода [63]. Что во многом схоже с эндокринными факторами невынашивания беременности.

Для восполнения дефицита 2 фазы используют экзогенный прогестерон в различных формах. Снижение уровня прогестерона наблюдается у женщин с рецидивирующей потерей беременности. Аномалии лютеиновой фазы встречаются у $35 \%$ женщин с рецидивирующей потерей беременности $[32,64]$. В связи с одной из теорий возникновения снижения рецептивности эндометрия из-за децидуального воспаления прогестерон рассматривают гормональным медиатором, дисрегуляция которого активирует промежуточные сигнальные пути, которые могут бытьоднимизведущимзвеномразвитияэтогопроцесса[65]. Согласно Dumesic DA и соавт. женщины с СПЯ имеют в 2,7 раза выше риск развития рака эндометрия из-за длительного воздействие на эндометрии гиперэстрогении из-за хронический ановуляции и резистентности эндометрия к прогестерону. А также частота встречаемости аномальных маточных кровотечений и гиперплазии эндометрия у пациенток с СПЯ следует терапевтически компенсировать с помощью КОК или непрерывного режима микронизированного прогестагена, или внутриматочной системой с левоноргестрелом [66]. Вагинальный прогестерон лучше увеличивает рецептивность эндометрия, т.к. только приблизительно 10\% перорального прогестерона абсорбируется через желудочно-кишечный тракт, а процент беременности ниже в циклах ВРТ, в которых использовали пероральный прогестерон по сравнению с вагинальной или внутримышечной формой [67]. Клинические эффекты микронизированного прогестерона аналогичны эндогенному прогестерону: прогестагенный эффект, антиэстрогенный эффект, антиальдостероновый эффект, антиандрогенный эффект (физиологический эффект регуляции уровня андрогенов), токолитический эффект, успокаивающий эффект, положительный нейротропный эффект [63].

В настоящее время пациенткам с ожирением и в частности с СПЯ, может быть рекомендовано использовать микронизированный прогестерон вагинально как на предгравидарном этапе, так и во время беременности. Согласно данным прямых сравнительных исследований, дидрогестерон не имеет преимуществ в клинической эффективности перед микронизированным прогестероном в поддержке лютеиновой фазы и при угрожающем выкидыше [68, 69]. Угроза прерывания беременности тяжелое психо-эмоциональное состояние для любой женщины. Угроза прерывания беременности отрицательно воздействует на психическое состояние женщин (повышается тревожность и уровень депрессии). Для некоторых 
женщин психологическая травма оказывается достаточно серьезной и продолжительной. Этот факт должен приниматься во внимание специалистами, оказывающими помощь таким пациенткам [70]. Следует отметить, что анксиолитическое действие, зафиксированное только у микронизированного прогестерона, сможет способствовать снижению тревоги и расстройства настроения в такой ситуации [71].

Согласно исследованию 2018 года прогестерон и родственные молекулы могут быть использованы в будущем при:

- лечении острого нарушения мозгового кровообращения,

реабилитации при черепно-мозговой травме,

терапии демениализирующих заболеваний,

болезни Альцгеймера [72].

Избыточный вес и ожирение способны серьезно повышать онкориски, в частности риск рака эндометрия и яичников [73]. Учитывая взаимосвязь ИМТ и рака органовполовой системы, можно предположить, что мероприятия по снижению веса могут снизить риск возникновения, заболеваемости и смертности от этого серьезного заболевания. В исследовании, посвященном выживаемости после рака эндометрия, 60\% опрошенных были заинтересованы в участии в мероприятиях по контролю за весом [74]. Бариатрическая хирургия также может помочь снизить риск некоторых видов гинекологических заболеваний. Например, было обнаружено, что показатели рака эндометрия значительно ниже среди женщин после бариатрических операций по сравнению с участницами, не подвергшимися хирургическому вмешательству [75]. Следует отметить, что изменение гормонального статуса у пациенток с ожирением может провоцировать онкориски. Именно баланс между уровнями эстрогена и прогестереона играет лючевую роль, как считает большинство авторов, в развитии рака эндометрия при ожирении: прогестерон вызывает дифференцировку, остановку клеточного цикла и апоптоз в эндометрии, действуя как настоящий супрессор опухоли, а при ожирении возникает хроническая ановуляция и, следовательно, уменьшение прогестерона, повышение непропорционально эстрогеновой активности. В связи с этим фактом, ассоциация между ожирением и ановуляцией имеет значительный риск развития рака эндометрия [76-78]. Стоит отметить, что ассоциация уровня прогестерона и заболеваемости раком эндометрия начинается у девочки уже с этапа менархе. Любое нарушение соотношения эстрогена/прогестерон будет в т.ч. долгосрочным фактором риска развития гиперплазии эндометрия и рака эндометрия. Основной детерминантой будет являться степень и продолжительность прогестерон-дефицитного состояния [56]. Крайне важно следить за ИМТ у девочки-подростка уже с этого периода.

Таким образом, высокий ИМТ повышает риск репродуктивных нарушений, таких как СПЯ, ГСД и онкологические заболевания. Ожирение повышает риск неблагоприятных исходов беременности для матери и плода. Программы по снижению веса и консультации диетологов обязательно должны внедрятся в гинекологические алгоритмы и рассматриваться как неотъемлемая часть лечения женщин, сталкивающихся с проблемой лишнего веса. Необходимо динамическое наблюдение за пациентками и контроль неблагоприятного воздействия СПЯ, раковых заболеваний или чрезмерной прибавки веса во время беременности на пищевое поведение, психологическую сферу для того, чтобы вовремя направить к соответствующим специалистам в области психического здоровья. Материнское ожирение и чрезмерное потребление пищи приводят к повышенному риску ожирения и метаболических и сердечно-сосудистых заболеваний у потомства. Внутриутробная среда зависит от питания матери и определяет не только риск возникновения заболевания, но и момент его начала и интенсивность патологического процесса. Для профилактики хронических заболеваний важно обучать беременных принципам рационального питания и образа жизни, а также информировать о возможных неблагоприятных последствиях неправильного питания для здоровья их детей.

\section{ДОПОЛНИТЕЛЬНАЯ ИНФОРМАЦИЯ}

Источник финансирования. Работа выполнена в рамках Государственного задания №AАAАА18-118051590062-6 «Разработка методов ведения пациентов с эндокринопатиями и бесплодием с учетом постнатальных исходов в программах вспомогательных репродуктивных технологий».

Конфликт интересов. Авторы декларируют отсутствие явных и потенциальных конфликтов интересов, связанных с публикацией настоящей статьи.

Участие авторов: Концепция и дизайн исследования - Абсатарова Ю.С., Андреева Е.Н. Сбор и обработка материала - Фурсенко В.А., Шереметьева Е.В. Написание текста - Абсатарова Ю.С., Шереметьева Е.В. Редактирование - Андреева Е.Н. Все авторы внесли значимый вклад в проведение исследования и подготовку статьи, прочли и одобрили финальную версию статьи перед публикацией.

\section{СПИСОК ЛИТЕРАТУРЫ | REFERENCES}

1. Who.int [Internet]. Всемирная Организация Здравоохранения. Центр СМИ. Информационный бюллетень «Ожирение и избыточный вес». [updated 2018 Feb 16; cited 2019 Jan 28]. Available from: https:// www.who.int/news-room/fact-sheets/detail/obesity-and-overweight

2. Who.int [Internet]. World Health Organisation Global Health Observatory data repository. Prevalence of obesity among adults, BMI $\geq 30$, age-standardized. Estimates by country. [updated $2017 \mathrm{Sep} 22$; cited 2019 Jan 28]. Available from: http://apps.who.int/gho/data/node. main.A900A?lang=en

3. Rachoń D, Teede H. Ovarian function and obesity —-Interrelationship, impact on women's reproductive lifespan and treatment options. Mol Cell Endocrinol. 2010;316(2):172-179. doi: 10.1016/.jmce.2009.09.026

4. Moran $\sqcup$, Norman RJ, Teede HJ. Metabolic risk in PCOS: phenotype and adiposity impact. Trends Endocrinol Metab. 2015;26(3):136-143. doi: 10.1016/j.tem.2014.12.003
5. Setji TL, Brown AJ. Polycystic Ovary syndrome: Update on diagnosis and treatment. Am J Med. 2014. doi: 10.1016/j.amjmed.2014.04.017

6. Dokras A. Mood and anxiety disorders in women with PCOS. Steroids. 2012;77(4):338-341. doi: 10.1016/j.steroids.2011.12.008

7. Karacan E, Caglar GS, Gürsoy AY, Yilmaz MB. Body Satisfaction and Eating Attitudes among Girls and Young Women with and without Polycystic Ovary Syndrome. J Pediatr Adolesc Gynecol. 2014;27(2):72-77. doi: 10.1016/j.jpag.2013.08.003

8. Lee I, Cooney LG, Saini S, et al. Increased risk of disordered eating in polycystic ovary syndrome. Fertil Steril. 2017;107(3):796-802. doi: 10.1016/j.fertnstert.2016.12.014

9. Larsson I, Hulthén L, Landén $M$, et al. Dietary intake, resting energy expenditure, and eating behavior in women with and without polycystic ovary syndrome. Clin Nutr. 2016;35(1):213-218. doi: 10.1016/j.clnu.2015.02.006 
10. Butterworth J, Deguara J, Borg C-M. Bariatric Surgery, Polycystic Ovary Syndrome, and Infertility. J Obes. 2016;2016:1-6. doi: 10.1155/2016/1871594

11. Teede HJ, Misso ML, Costello MF, et al. Recommendations from the international evidence-based guideline for the assessment and management of polycystic ovary syndrome. Hum Reprod. 2018:33(9):1602-1618. doi: 10.1093/humrep/dey256

12. Foroozanfard F, Rafiei $\mathrm{H}$, Samimi M, et al. The effects of dietary approaches to stop hypertension diet on weight loss, anti-Müllerian hormone and metabolic profiles in women with polycystic ovary syndrome: A randomized clinical trial. Clin Endocrinol (Oxf). 2017;87(1):51-58. doi: 10.1111/cen.13333

13. Guenard $F$, Deshaies $Y$, Cianflone $K$, et al. Differential methylation in glucoregulatory genes of offspring born before vs. after maternal gastrointestinal bypass surgery. Proc Natl Acad Sci. 2013;110(28):11439-11444. doi: 10.1073/pnas.1216959110

14. Lim SS, Davies MJ, Norman RJ, Moran LJ. Overweight, obesity and central obesity in women with polycystic ovary syndrome: a systematic review and meta-analysis. Hum Reprod Update. 2012;18(6):618-637. doi: 10.1093/humupd/dms030

15. van der Steeg JW, Steures P, Eijkemans MJC, et al. Obesity affects spontaneous pregnancy chances in subfertile, ovulatory women. Hum Reprod. 2007;23(2):324-328. doi: 10.1093/humrep/dem371

16. Moran $\sqcup$, Noakes M, Clifton PM, et al. Dietary Composition in Restoring Reproductive and Metabolic Physiology in Overweight Women with Polycystic Ovary Syndrome. J Clin Endocrinol Metab. 2003;88(2):812-819. doi: 10.1210/jc.2002-020815

17. Gesink Law DC, Maclehose RF, Longnecker MP. Obesity and time to pregnancy. Hum Reprod. 2006;22(2):414-420. doi: 10.1093/humrep/del400

18. Shah DK, Missmer SA, Berry KF, et al. Effect of Obesity on Oocyte and Embryo Quality in Women Undergoing In Vitro Fertilization. Obstet Gynecol. 2011;118(1):63-70. doi: 10.1097/AOG.0b013e31821fd360

19. Luke B, Brown MB, Stern JE, et al. Female obesity adversely affects assisted reproductive technology (ART) pregnancy and live birth rates. Hum Reprod. 2011;26(1):245-252. doi: 10.1093/humrep/deq306

20. Institute of Medicine and National Research Council. Weight Gain During Pregnancy: Reexamining the Guidelines. Washington, DC: The National Academies Press; 2009. doi: 10.17226/12584

21. Restall A, Taylor RS, Thompson JMD, et al. Risk Factors for Excessive Gestational Weight Gain in a Healthy, Nulliparous Cohort. J Obes. 2014:2014:1-9. doi: 10.1155/2014/148391

22. Deputy NP, Sharma AJ, Kim SY, Hinkle SN. Prevalence and Characteristics Associated With Gestational Weight Gain Adequacy. Obstet Gynecol. 2015;125(4):773-781 doi: 10.1097/AOG.0000000000000739

23. Margerison Zilko CE, Rehkopf D, Abrams B. Association of maternal gestational weight gain with short- and long-term maternal and child health outcomes. Am J Obstet Gynecol. 2010;202(6):574.e1-574.e8. doi: 10.1016/j.ajog.2009.12.007

24. Silveira ML, Ertel KA, Dole N, Chasan-Taber L. The role of body image in prenatal and postpartum depression: a critical review of the literature. Arch Womens Ment Health. 2015;18(3):409-421. doi: 10.1007/s00737-015-0525-0

25. Skouteris $\mathrm{H}$, Carr R, Wertheim EH, et al. A prospective study of factors that lead to body dissatisfaction during pregnancy. Body Image. 2005:2(4):347-361. doi: 10.1016/j.bodyim.2005.09.002

26. Boudet-Berquier J, Salanave B, Desenclos J-C, Castetbon K. Sociodemographic factors and pregnancy outcomes associated with prepregnancy obesity: effect modification of parity in the nationwide Epifane birth-cohort. BMC Pregnancy Childbirth. 2017;17(1):273. doi: 10.1186/s12884-017-1456-8

27. Waddington CH. The Epigenotype. Int J Epidemiol. 2012;41(1):10-13. doi: 10.1093/ije/dyr184

28. Carolan-Olah M, Duarte-Gardea M, Lechuga J. A critical review: early life nutrition and prenatal programming for adult disease. J Clin Nurs. 2015;24(23-24):3716-3729. doi: 10.1111/jocn.12951

29. Yan X, Huang Y, Zhao J-X, et al. Maternal obesity downregulates microRNA let-7g expression, a possible mechanism for enhanced adipogenesis during ovine fetal skeletal muscle development. Int J Obes. 2013;37(4):568-575. doi: 10.1038/ijo.2012.69

30. Frias A, Grove K. Obesity: A Transgenerational Problem Linked to Nutrition during Pregnancy. Semin Reprod Med. 2012;30(06):472-478. doi: 10.1055/s-0032-1328875

31. Dabelea D, Crume T. Maternal Environment and the Transgenerational Cycle of Obesity and Diabetes. Diabetes. 2011;60(7):1849-1855. doi: 10.2337/db11-0400
32. Dante $G$, Vaccaro V, Facchinetti F. Use of progestagens during early pregnancy. Facts Views Vis Obgyn. 2013;5(1):66-71. PMID: 24753930.

33. Hou M, Chu Z, Liu T, et al. A high-fat maternal diet decreases adiponectin receptor-1 expression in offspring. J Matern Neonatal Med. 2015;28(2):216-221. doi: 10.3109/14767058.2014.914489

34. Fan L, Lindsley SR, Comstock SM, et al. Maternal high-fat diet impacts endothelial function in nonhuman primate offspring. Int J Obes. 2013;37(2):254-262. doi: 10.1038/ijo.2012.42

35. Ornoy A. Prenatal origin of obesity and their complications: Gestational diabetes, maternal overweight and the paradoxical effects of fetal growth restriction and macrosomia. Reprod Toxicol. 2011;32(2):205-212. doi: 10.1016/j.reprotox.2011.05.002

36. Chen C-P. Prenatal findings and the genetic diagnosis of fetal overgrowth disorders: Simpson-Golabi-Behmel syndrome, Sotos syndrome, and Beckwith-Wiedemann syndrome. Taiwan J Obstet Gynecol. 2012;51(2):186-191. doi: 10.1016/j.tjog.2012.04.004

37. Marchi J, Berg M, Dencker A, Olander EK, Begley C. Risks associated with obesity in pregnancy, for the mother and baby: a systematic review of reviews. Obes Rev. 2015;16(8):621-638. doi: 10.1111/obr.12288

38. $\mathrm{XuJ}$, Ye J, Wu Y, et al. Reduced Fetal Telomere Length in Gestational Diabetes. Frasch MG, ed. PLoS One. 2014;9(1):e86161. doi: 10.1371/journal.pone.0086161

39. Ryckman K, Smith C. Epigenetic and developmental influences on the risk of obesity, diabetes, and metabolic syndrome. Diabetes, Metab Syndr Obes Targets Ther. 2015;2015(8):295-302. doi: 10.2147/DMSO.S61296

40. Monk M, Boubelik M, Lehnert S. Temporal and regional changes in DNA methylation in the embryonic, extraembryonic and germ cell lineages during mouse embryo development. Development. 1987;99(3):371-382. PMID: 3653008

41. Cardozo E, Pavone ME, Hirshfeld-Cytron JE. Metabolic syndrome and oocyte quality. Trends Endocrinol Metab. 2011;22(3):103-109. doi: 10.1016/j.tem.2010.12.002

42. Zambrano E, Martínez-Samayoa PM, Bautista CJ, et al. Sex differences in transgenerational alterations of growth and metabolism in progeny ( $F 2$ ) of female offspring (F1) of rats fed a low protein diet during pregnancy and lactation. J Physiol. 2005;566(1):225-236. doi: 10.1113/jphysiol.2005.086462

43. Timmermans S, Steegers-Theunissen RP, Vujkovic M, et al. The Mediterranean diet and fetal size parameters: the Generation R Study. Br J Nutr. 2012;108(08):1399-1409. doi: 10.1017/S000711451100691X

44. Lillycrop KA, Phillips ES, Jackson AA, Hanson MA, Burdge GC. Dietary Protein Restriction of Pregnant Rats Induces and Folic Acid Supplementation Prevents Epigenetic Modification of Hepatic Gene Expression in the Offspring. J Nutr. 2005;135(6):1382-1386. doi: 10.1093/jn/135.6.1382

45. Cawley S, Mullaney L, McKeating A, Farren M, McCartney D, Turner MJ. A review of European guidelines on periconceptional folic acid supplementation. Eur J Clin Nutr. 2016;70(2):143-154. doi: 10.1038/ejcn.2015.131

46. Bailey LB, Stover PJ, McNulty H, et al. Biomarkers of Nutrition for Development-Folate Review. J Nutr. 2015;145(7):1636S-1680S. doi: 10.3945/jn.114.206599

47. Mojtabai R. Body mass index and serum folate in childbearing age women. Eur J Epidemiol. 2004;19(11):1029-1036. doi: 10.1007/s10654-004-2253-z

48. Knight BA, Shields BM, Brook A, et al. Lower Circulating B12 Is Associated with Higher Obesity and Insulin Resistance during Pregnancy in a Non-Diabetic White British Population. Sengupta S, editor. PLoS One. 2015;10(8):e0135268. doi: 10.1371/journal.pone.0135268

49. Shen M, Chaudhry SH, MacFarlane AJ, et al. Serum and redblood-cell folate demonstrate differential associations with BMI in pregnant women. Public Health Nutr. 2016;19(14):2572-2579. doi: $10.1017 / S 1368980016000756$

50. Dean SV., Lassi ZS, Imam AM, Bhutta ZA. Preconception care: nutritional risks and interventions. Reprod Health. 2014;11(Suppl 3):S3. doi: 10.1186/1742-4755-11-S3-S3

51. Blode H, Diefenbach, Trummer, et al. EE-drospirenone-levomefolate calcium versus EE-drospirenone + folic acid: folate status during 24 weeks of treatment and over 20 weeks following treatment cessation. Int J Womens Health. 2013;2013(5):149-163. doi: 10.2147/IJWH.S37254

52. Castaño PM, Aydemir A, Sampson-Landers C, Lynen R. The folate status of reproductive-aged women in a randomised trial of a folate-fortified oral contraceptive: dietary and blood assessments. Public Health Nutr. 2014;17(06):1375-1383. doi: 10.1017/ S1368980013000864 
53. Pfeifer S, Fritz M, Goldberg J, et al. The clinical relevance of luteal phase deficiency: a committee opinion. Fertil Steril. 2012;98(5):11121117. doi: 10.1016/j.fertnstert.2012.06.050

54. Jain A, Polotsky AJ, Rochester D, et al. Pulsatile Luteinizing Hormone Amplitude and Progesterone Metabolite Excretion Are Reduced in Obese Women. J Clin Endocrinol Metab. 2007;92(7):2468-2473. doi: 10.1210/jc.2006-2274

55. Vannuccini S, Clifton VL, Fraser IS, et al. Infertility and reproductive disorders: impact of hormonal and inflammatory mechanisms on pregnancy outcome. Hum Reprod Update. 2016;22(1):104-115. doi: 10.1093/humupd/dmv044

56. Schindler AE. Progestogen deficiency and endometrial cancer risk Maturitas. 2009;62(4):334-337. doi: 10.1016/j.maturitas.2008.12.018

57. Nawaz FH, Khalid R, Naru T, Rizvi J. Does continuous use of metformin throughout pregnancy improve pregnancy outcomes in women with polycystic ovarian syndrome? J Obstet Gynaecol Res. 2008;34(5):832-837. doi: 10.1111//j.1447-0756.2008.00856.x

58. Costello MF, Eden JA. A systematic review of the reproductive system effects of metformin in patients with polycystic ovary syndrome. Fertil Steril. 2003;79(1):1-13. doi: 10.1016/S0015-0282(02)04554-5

59. Jakubowicz DJ, luorno MJ, Jakubowicz S, et al. Effects of Metformin on Early Pregnancy Loss in the Polycystic Ovary Syndrome. J Clin Endocrinol Metab. 2002;87(2):524-529. doi: 10.1210/jcem.87.2.8207

60. Ben-Haroush A, Yogev Y, Hod M. Epidemiology of gestational diabetes mellitus and its association with Type 2 diabetes. Diabet Med. 2004;21(2):103-113. doi: 10.1046/j.1464-5491.2003.00985.x

61. Rai R, Backos M, Rushworth F, Regan L. Polycystic ovaries and recurrent miscarriage - a reappraisal. Hum Reprod. 2000;15(3):612-615. doi: 10.1093/humrep/15.3.612

62. Glueck CJ, Wang P, Goldenberg N, Sieve-Smith L. Pregnancy outcomes among women with polycystic ovary syndrome treated with metformin. Hum Reprod. 2002;17(11):2858-2864. doi: 10.1093/humrep/17.11.2858

63. Макацария А.Д., Передеряева Е.Б., Пшеничникова Т.Б. Особенности применения натурального прогестерона в профилактике осложнений беременности у женщин с метаболическим синдромом // Эффективная фармакотерапия. - 2008. - №5. - С.11-14. [Makatsariya AD, Perederyaeva EB, Pshenichnikova TB. Osobennosti primeneniya natural'nogo progesterona $v$ profilaktike oslozhnenii beremennosti u zhenshchin s metabolicheskim sindromom. Ėffektivnaâ farmakoterapiâ. 2008;(5):11-14. (in Russ.)]

64. Arredondo F, Noble LS. Endocrinology of Recurrent Pregnancy Loss. Semin Reprod Med. 2006;24(01):033-039. doi: 10.1055/s-2006-931799

65. Norwitz ER, Bonney EA, Snegovskikh VV., et al. Molecular Regulation of Parturition: The Role of the Decidual Clock. Cold Spring Harb Perspect Med. 2015;5(11):a023143. doi: 10.1101/cshperspect.a023143

66. Dumesic DA, Lobo RA. Cancer risk and PCOS. Steroids. 2013;78(8):782 785. doi: 10.1016/j.steroids.2013.04.004
67. Daya S, Gunby J. Luteal phase support in assisted reproduction cycles. In: Daya S, editor. Cochrane Database of Systematic Reviews. Chichester, UK: John Wiley \& Sons, Ltd; 2004. doi: 10.1002/14651858.CD004830

68. Salehpour S, Tamimi M, Saharkhiz N. Comparison of oral dydrogesterone with suppository vaginal progesterone for luteal-phase support in in vitro fertilization (IVF): A randomized clinical trial. Iran J Reprod Med. 2013;11(11):913-918. PMID: 24639716

69. Tomic V, Tomic J, Klaic DZ, Kasum M, Kuna K. Oral dydrogesterone versus vaginal progesterone gel in the luteal phase support: randomized controlled trial. Eur J Obstet Gynecol Reprod Biol. 2015;186:49-53. doi: 10.1016/j.ejogrb.2014.11.002

70. Адамян Л.В., Артымук Н.В., Белокриницкая Т.В., и др. Выкидыш в ранние сроки беременности: диагностика и тактика ведения. Клинические рекомендации (протокол лечения). - М.: Российское общество акушеров-гинекологов, 2016. [Adamyan LV, Artymuk NV, Belokrinitskaya TV, et al. Vykidysh v rannie sroki beremennosti: diagnostika i taktika vedeniya. Klinicheskie rekomendatsii (protokol lecheniya). Moscow: Rossiiskoe obshchestvo akusherov-g inekologov; 2016. (In Russ.)]

71. Dennerstein L, Spencer-Gardner C, Gotts G, et al. Progesterone and the premenstrual syndrome: a double blind crossover trial. BMJ. 1985;290(6482):1617-1621. doi: 10.1136/bmj.290.6482.1617

72. Sitruk-Ware R. Non-clinical studies of progesterone. Climacteric. 2018;21(4):315-320.

doi: 10.1080/13697137.2018.1463982

73. Keum N, Greenwood DC, Lee DH, et al. Adult Weight Gain and Adiposity-Related Cancers: A Dose-Response Meta-Analysis of Prospective Observational Studies. JNCI J Nat/ Cancer Inst. 2015;107(2):djv088. doi: 10.1093/jnci/djv088

74. Haggerty AF, Huepenbecker S, Sarwer DB, et al. The use of novel technology-based weight loss interventions for obese women with endometrial hyperplasia and cancer. Gynecol Oncol. 2016; 140(2):239-244. doi: 10.1016/j.ygyno.2015.11.033

75. Anveden $\AA$, Taube M, Peltonen M, et al. Long-term incidence of female-specific cancer after bariatric surgery or usual care in the Swedish Obese Subjects Study. Gynecol Oncol. 2017;145(2):224-229. doi: 10.1016/j.ygyno.2017.02.036

76. Benedetto C, Salvagno F, Canuto EM, Gennarelli G. Obesity and female malignancies. Best Pract Res Clin Obstet Gynaecol. 2015;29(4):528-540. doi: 10.1016/j.bpobgyn.2015.01.003

77. Yang S, Thiel KW, Leslie KK. Progesterone: the ultimate endometrial tumor suppressor. Trends Endocrinol Metab. 2011;22(4):145-152. doi: 10.1016/j.tem.2011.01.005

78. Gottschau M, Kjaer SK, Jensen A, Munk C, Mellemkjaer L. Risk of cancer among women with polycystic ovary syndrome: A Danish cohort study. Gynecol Oncol. 2015;136(1):99-103. doi: 10.1016/j.ygyno.2014.11.012

\section{ИНФОРМАЦИЯ ОБ АВТОРАХ [AUTHORS INFO]:}

*Абсатарова Юлия Сергеевна, к.м.н. [Yulia S. Absatarova, MD, PhD]; адрес: Россия, 117036, Москва, улица Дм. Ульянова, д.11 [address: 11 Dm. Ulyanova street, 117036 Moscow, Russia]; ORCID: https://orcid.org/0000-0003-0696-5367; eLibrary SPIN: 2220-9464; e-mail: korsil2008@yandex.ru

Андреева Елена Николаевна, д.м.Н., професcop. [Elena N. Andreeva MD, PhD, Professor]; ORCID: https://orcid.org/0000-0001-8425-0020, eLibrary SPIN: 1239-2937; e-mail: endogin@mail.ru Шереметьева Екатерина Викторовна, к.м.н. [Ekaterina V. Sheremetyeva, MD, PhD]; ORCID: https://orcid.org/0000-0001-7177-0254; eLibrary SPIN: 9413-5136; e-mail: s1981k@yandex.ru Фурсенко Валентина Алексеевна, аспирант [Valentina A. Fursenko, MD, postgraduate student]; ORCID: https://orcid.org/0000-0003-0517-8687, eLibrary SPIN: 7733-1781; e-mail: dr.v1@bk.ru

\section{ЦИТИРОВАТЬ:}

Андреева Е.Н., Абсатарова Ю.С., Шереметьева Е.В., Фурсенко В.А. Ожирение и репродуктивная функция у женщин: эпигенетический и сомато-психологические особенности // Ожирение и метаболизм. — 2019. — Т.16. — №2. C.9-15. doi: https://doi.org/10.14341/omet10113

\section{TO CITE THIS ARTICLE:}

Andreeva EN, Absatarova YU, Sheremetyeva EV, Fursenko VA. Obesity and reproductive function of women: epigenetic and somato-psychological features. Obesity and metabolism. 2019;16(2):9-15. doi: https://doi.org/10.14341/omet10113 